disruptions to this bacterial community are often associated with various diseases' progression, including gastrointestinal infection. One significant diarrheal pathogen is Vibrio species, the critical etiological agent to cholera and gastrointestinal infection that affects millions of people annually. They invade the host's gastrointestinal system, releasing virulence toxins that aid in the colonization, dysbiosis of the gut flora, and cause diarrhea. The gut microbiota community is diverse, yet how this microbial diversity confers resistance or susceptibility to intestinal pathogens is not well explored. Hence, this systematic review aims to understand the gut microbiota variation in response to Vibrio infection.

Methods This systematic review was conducted based on the PRISMA guidelines. Predefined MeSH terms 'Vibrio' AND 'gut microbiota' OR 'microbiome' were used in three databases (Pubmed, Ovid Medline, ProQuest; from database inception to June 2021). Studies reporting on the gut microbiota variation and Vibrio were included. Studies without Vibrio and/or gut microbiome data were excluded, including conference proceedings, reviews, systematic reviews, meta-analyses, and commentaries.

Results Six studies according to the study's inclusion criteria out of 243 articles were selected for qualitative analysis. Three studies identified the gut microbiota composition in patients' samples with Vibrio species infection, while the remaining studies explored the modulation of gut microbiota in Vibrioinduced animal models. The human studies demonstrated variation in the gut microbiome of patients with Vibrio infection as compared to the healthy subjects. Bacteroides, Lactobacillus, Bifidobacterium, and Akkermansia are among the prominent taxa found to be significantly decreased in abundance but increase in the taxa of Firmicutes among the patients and animals, elevating the infection and causing diarrhea. An in vivo study also reported the abundance of Ruminococcus/Blautia obeum, that able to inhibit the Vibrio cholerae virulence in the gut (IDDF2021-ABS-0126 Figure 1. Illustration of gut microbiota variation in response to Vibrio infection).

Conclusions There is a potential connection between gut dysbiosis and Vibrio species infection, in which the gut microbiota possibly increase/decrease its abundance to inhibit the infection. These findings provide insights on gut microbiota response towards environmental pathogen and frame proper treatment strategies in clinical settings.

\section{IDDF2021-ABS-0130 DYSBIOSIS OF GUT ARCHAEA IN OBESITY RECOVERED AFTER BARIATRIC SURGERY}

${ }^{1}$ Keli Yang*, ${ }^{2}$ Jia Yang, ${ }^{3}$ Jingwan Zhang, ${ }^{2}$ Qichun Wei, ${ }^{3}$ Siew Chien Ng, ${ }^{1}$ Hui Wang ${ }^{1}$ Department of Colorectal Surgery, Guangdong Provincial Key Laboratory of Colorectal and Pelvic Floor Diseases, The Sixth Affiliated Hospital of Sun Yat-sen University, Guangzhou, China; ${ }^{2}$ Department of Radiation Oncology, Key Laboratory of Cancer Prevention and Intervention, Ministry of Education, The Second Affiliated Hospital, Zhejiang University School of Medicine, Hangzhou, China; ${ }^{3}$ Center for Gut Microbiota Research, Department of Medicine and Therapeutics, Institute of Digestive Disease, State Key Laboratory of Digestive Diseases, The Chinese University of Hong Kong, Hong Kong

\subsection{6/gutjnl-2021-IDDF.43}

Background Obesity is associated with altered gut microbiota. Archaeome is an important part in intestinal microbiota, however, the role of archaea in obesity remains unknown. We aimed to delineate the alterations of gut archaea in subjects with obesity and explore the effect of bariatric surgery-associated weight loss intervention in modulating the gut archaeal composition.
Methods A total of 231 obese subjects and 184 lean controls with shotgun metagenomic sequencing available were retracted from three public cohorts. Of which, 23 obese subjects undergone bariatric surgery were followed up for 3 months. Alterations of gut archaeome, bacteriome and archaeal-bacterial correlations were assessed in obese and lean subjects.

Results Fecal archaea microbiota of obese subjects showed a significantly lower Chao1 richness and Shannon diversity compared to lean controls $(p<0.05)$. Principal component analysis of archaeome revealed distinct clusters in obese subjects and lean controls $(p<0.05)$. Two archaeal orders including Methanosarcinales and Methanococcales were increased and three orders including Natrialbales, Methanocellales and Thermoproteales were decreased in obese subjects compared with lean controls. Top 20 differentially abundant archaeal genera discriminated obese subjects from lean controls with an area under the receiver operating characteristic curve (AUC) of 0.79 (95\% CI $0.72-0.85)$ in the discovery cohort. It was further validated in two independent cohorts, with AUC of 0.83 (0.73-0.92) and 0.86 (0.77-0.94), respectively. Further prediction analysis by archaeal genera revealed a significantly lower probability of obesity in subjects after bariatric surgery than their baseline $(p<0.05)$. Moreover, the ecological analysis showed significantly lower trans-kingdom correlations between archaea and bacteria in obese subjects compared with lean controls, and these correlations were partly recovered in subjects receiving bariatric surgery.

Conclusions This study demonstrated for the first time that obesity is characterized by gut archaeome dysbiosis across multiple cohorts with different demographic characteristics. Weight loss by bariatric surgery-induced a significant change of gut archaeome, suggesting that gut archaeome may play an important role in treating obesity. Studies are needed to explore the potential benefit of reshaping gut archaeome to improve weight loss intervention efficacy.

\section{IDDF2021-ABS-0132 MOVING BEYOND THE GASTROINTESTINAL TRACT: THE INVOLVEMENT OF GUT MICROBIOME IN ENDOMETRIOSIS}

${ }^{1}$ Hooi-Leng Ser*, ${ }^{2}$ Joshua You Jing Wong, ${ }^{1}$ Vengadesh Letchumanan, ${ }^{1} J o d i$ Woan-Fei Law, ${ }^{3}$ Loh Teng-Hern Tan, ${ }^{1}$ Learn-Han Lee. ${ }^{1}$ Novel Bacteria and Drug Discovery (NBDD) Research Group, Microbiome and Bioresource Research Strength, Jeffrey Cheah School of Medicine and Health Sciences, Monash University Malaysia, Malaysia; ${ }^{2}$ West Hertfordshire Hospitals, NHS Trust, Vicarage Rd, Watford, Hertfordshire, UK; ${ }^{3}$ Clinical School Johor Bahru, Jeffrey Cheah School of Medicine and Health Sciences, Monash University Malaysia, Malaysia

\subsection{6/gutjnl-2021-IDDF.44}

Background The importance of gut health and microbiome have been emphasized in women's health. Endometriosis is characterized by abnormal growth of endometrial cells that occurs outside of the uterus (IDDF2021-ABS-0132 Figure 1. Potential association of gut microbiome in endometriosis). Animal models of endometriosis display different gut microbiota compositions compared to healthy controls, and the oral administration of broad-spectrum antibiotics has been shown to reduce disease progression. The current study investigates the relationship between the gut microbiome and endometriosis in humans before exploring potential mechanisms involved. Methods Searches were performed in three databases (PubMed, Ovid Medline, ScienceDirect; database inception to 31st May 2021 using 'gut', 'microbiome,' or 'microbiota' combined with 'endometriosis' following PRISMA guidelines). All 


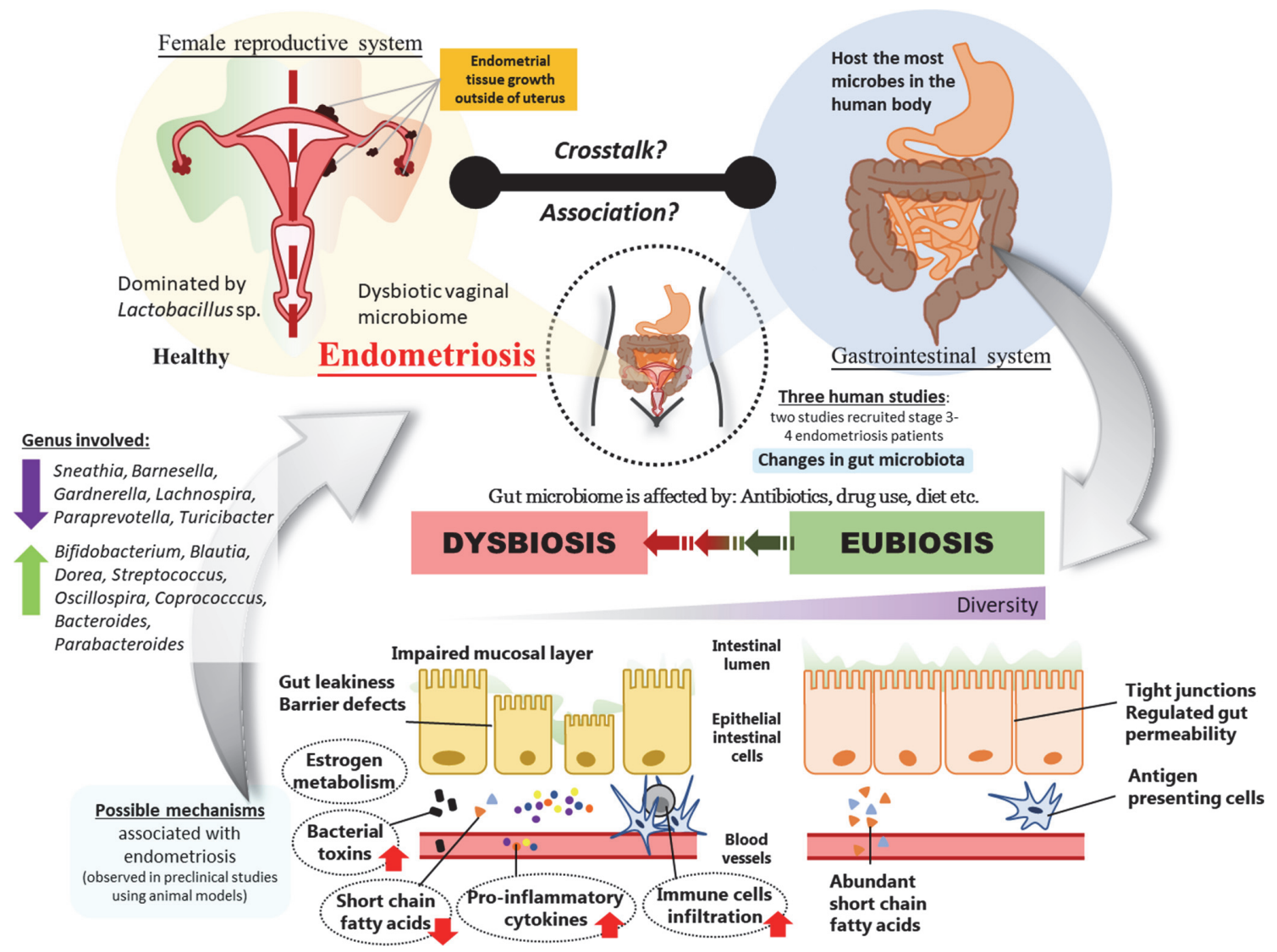

Abstract IDDF2021-ABS-0132 Figure 1

titles and abstracts retrieved were screened based on the inclusion and exclusion criteria. Studies reporting gut microbiome data in relation to endometriosis in humans were included in the current analysis but not those without gut microbiome data.

Results Three studies were selected for qualitative analysis according to the inclusion and exclusion criteria. All studies investigated the gut microbiota composition by whole genome sequencing, specifically targeting the $16 \mathrm{~S}$ rRNA gene. These studies detected significant differences in selected bacterial abundance in the gut microbiome among endometriosis patients and control. Two of them discussed potential bacterial group(s) that correlates with gastrointestinal symptoms in endometriosis patients. A study in 2019 showed that two endometriosis patients had high Escherichia/Shigella in stool, and subsequent follow-up showed severe bowel involvement by endometriosis requiring segmental colon resection. Another team also indicated in their recent work that those with endometrial involvement of the gastrointestinal tract had a higher abundance of Lactococcus (Class Bacilli) compared to those without involvement. The same study noted a non-significant enrichment of Enterobacteriaceae in endometriosis patients that warrants further investigation to evaluate the involvement of gut microbiota in endometriosis.

Conclusions Further studies are required to establish the association and involvement of gut microbiota in endometriosis. The heterogeneity in study design (inclusion/exclusion criteria etc.) increases the difficulty in making a meaningful comparison. More information on diet and antibiotic drug use need to be collected to provide a better overview and insightful analysis of the gut microbiome in endometriosis.

\section{IDDF2021-ABS-0140 GUT MICROBIOTA SIGNIFICANTLY CORRELATE WITH BODY CONSTITUTION IN TRADITIONAL CHINESE MEDICINE}

${ }^{1}$ Haohui Liu*, ${ }^{2}$ Meihui Xu, ${ }^{2}$ Kai Yee Toh, ${ }^{3}$ Chun Wie Chong, ${ }^{2}$ Jeremy Fung Yen Lim, ${ }^{4} J$ onathan Wei Jie Lee. ${ }^{1}$ School of Computer Science, Carnegie Mellon University, USA; ${ }^{2}$ Asian Microbiome Library (AMILI), Singapore; ${ }^{3}$ School of Pharmacy, Monash University Malaysia, Malaysia; ${ }^{4}$ Division of Gastroenterology and Hepatology, National University Hospital, Singapore

\subsection{6/gutjil-2021-IDDF.45}

Background The gut microbiome plays an instrumental role in cardiovascular, metabolic and gastrointestinal health (Valdes et al. 2018). In Traditional Chinese Medicine (TCM), one's health status can be described by nine body constitutions (BCs) which are broadly classified as 'Neutral', indicating optimal health, or 'Unbalanced', indicating suboptimal health. Similar to the gut microbiome, BCs are strongly influenced by lifestyle and diet. We evaluated the gut microbial composition of Neutral, Qi-Deficiency (QD), Phlegm-dampness (PD) and Damp Heat (DH) subjects. The latter three are unbalanced BCs, with QD associated with heart disease (Zhu et al. 2017), PD with obesity and metabolic syndrome (Wang et al. 2013), and DH with gastrointestinal disease (Liang et al. 2020).

Methods Stool samples were collected from 211 healthy subjects and gut microbial composition was profiled using $16 \mathrm{~S}$ rRNA amplicon sequencing of the V3-V4 regions. BCs were determined using the Constitution in Chinese Medicine Questionnaire (Wang, 2005). Alpha diversity of QD, PD and DH was inferred and compared against that of Neutral using the 